\title{
Diagnostik des Trockenen Auges - Praxis und interdisziplinäre Aspekte
}

\section{J. Horwath-Winter}

Universitäts-Augenklinik Graz, Österreich
Eine erfolgreiche Behandlung von Patienten mit Benetzungsstörungen setzt die diagnostische Umsetzung der Kenntnisse über die Komplexität des Tränenfilms voraus. Die ausreichende Befeuchtung der Augenoberfläche ist einem Regelkreis unterworfen, an dem die Lider mit den MeibomDrüsen, der Blinkmechanismus, die Zellen der Augenoberfläche, die Tränendrüsen und die neuronalen und hormonellen Steuerungsfaktoren Einfluss nehmen.

Der Stellenwert einer ausführlichen Anamnese und Symptomerhebung ist unabdingbar. Beim Gespräch mit dem Patienten kann eine erste Inspektion der Haut sowie der Lidfunktion inklusive Blinkfrequenz erfolgen.

$\mathrm{Zu}$ jeder Untersuchung eines Siccapatienten gehören eine Sehprobe für Ferne und Nähe, die Überprüfung der Binokularität und eventuell eine Skiaskopie, um asthenopäische Beschwerden von Siccabeschwerden differenzieren zu können, da die Symptome unserer Patienten zwar richtungsweisend, jedoch meist nicht spezifisch sind.

Bei der Spaltlampenuntersuchung achtet man auf die regelmäßige Stellung der Lider und der Tränenpünktchen und auf vollständigen Lidschluss. Die Höhe des Tränenmeniskus gibt eine orientierende Auskunft über das Tränenvolumen. Detritus, Schleimfäden, schaumiges Sekret, konjunktivale Injektion unterschiedlicher Ausprägung und lidkantenparallele Bindehautfalten sind wichtige Hinweise für eine Benetzungsstörung. Subepitheliale Fibrose und Symblepharonbildung können erste Zeichen eines okulären Pemphigoids sein. Die Überprüfung der Lidkante mit Expression des Meibom-Drüsen-Sekrets und die Beurteilung der Qualität des Sekrets sind wesentlich für die Erkennung einer Dysfunktion der Meibom-Drüsen. Die Applikation von Fluorescein ermöglicht die Messung der Tränenfilmaufreißzeit, die bei allen Formen des Trockenen Auges reduziert sein kann. Außerdem können Epitheldefekte der Hornhaut sichtbar gemacht bzw. verdeutlicht werden.

Für die genaue Beurteilung der Bindehaut stehen uns Farbstoffe wie Bengalrosa und Lissamingrün zur Verfügung. Bei schon deutlich sichtbarer Oberflächenschädigung sollte das besser verträglichere Lissamingrün gewählt werden.

Die Messung des Tränenvolumens erfolgt mit dem Schirmer-Test. Um die Fähigkeit der Tränendrüsen, Sekret zu sezernieren, überprüfen zu können, sollte dieser primär ohne Lokalanästhesie durchgeführt werden. Bei der Interpretation von Tränenmengen muss immer bedacht werden, dass das Tränenvolumen die situativ bedingte Antwort eines Reflexbogens darstellt, welche sich nach der Oberflächenbeschaffenheit und nach der Stabilität des Tränenfilms richtet. Geringe Tränenvolumina können durch eine erhöhte Stabilität, größere Mengen aber auch durch eine vermehrte Schädigung der Augenoberfläche bedingt sein.

Bei stark reduzierter Tränenmenge ist als zusätzlicher Test der Schirmer-Test mit Reizung der nasalen Schleimhaut mit Hilfe eines Wattestäbchens hilfreich. Bei fehlendem Anstieg des Messwertes besteht eine erhöhte Wahrscheinlichkeit auf ein Sjögren-Syndrom.

In den letzten Jahren hat sich überdies gezeigt, dass Patienten mit Benetzungsstörungen auch bei normaler Tränensekretion und durchgängigen Tränenwegen eine verlangsamte Fluorescein-Clearance aufweisen können - besonders bei schlaffen Lidern, Bindehautfalten und Dysfunktion der Meibom-Drüsen. Bei stagnierendem Tränenfilm findet sich ein erhöhter Gehalt an Entzündungsprodukten im Tränenfilm, der für die Symptomatik der Patienten ausschlaggebend sein kann.

Bei stark reduzierter Tränensekretion, besonders bei niedrigem Schirmer-Test mit nasaler Reizung und bestehender Augenoberflächenschädigung der Hornhaut und/oder Bindehaut ist eine weitere Abklärung in Richtung Sjögren-Syndrom indiziert, da zusätzlich eine extraglanduläre Beteiligung mit Pericarditis, Pleuritis sowie Nephritis möglich ist. Überdies muss ein stark erhöhtes Lymphomrisiko der Patienten beachtet werden. Da für die zusätzliche Diagnostik Spezialuntersuchungen wie Speicheldrüsenszintigraphie und eine Biopsie der kleinen Lippenspeicheldrüsen erforderlich sind, ist die Zuweisung an eine Spezialabteilung empfehlenswert, um den Zeitpunkt der Diagnose nicht zu verlängern.

Bei ausgeprägter Meibom-Drüsen-Dysfunktion ist eine hautfachärztliche Untersuchung indiziert um Erkrankungen wie Rosacea, Psoriasis oder seborrhoische Dermatitis abzuklären.

Nach wie vor gibt es keinen Test, der als Goldstandard für die Diagnose des Trockenen Auges gelten könnte. Die einzelnen Befunde sind Mosaiksteine für ein Gesamtbild, welches individuell interpretiert werden muss und erst so die exakte Diagnose ermöglicht. 